\title{
EL COMERCIO HISPANO-ARGELINO Y EL MOVIMIENTO DEL PUERTO DE ARGEL EN 1865, A TRAVÉS DE UNA MEMORIA DEL CONSULADO DE ESPAÑA
}

por

JUAN BTA. VILAR

\section{NOTA PRELIMINAR}

En el Archivo del Ministerio de Asuntos Exteriores, Sección Correspondencia de Argel, leg. 1.819, se conserva una interesante memoria fechada en 28 de febrero de 1866, y remitida al ministro de Estado por el cónsul general de España en Argelia. Se trata del preceptivo informe consular enviado anualmente a Madrid sobre el movimiento del puerto de Argel, referido en el presente caso al año precedente de 1865 .

El autor del informe, Carlos Vidal, se titula "cónsul general interino», siéndolo, en efecto, por cese, meses antes, de don Vicente de Zugasti, sin duda el diplomático europeo a la sazón con mayor experiencia sobre los asuntos argelinos. Zugasti había asumido la representación española en Argel apenas meses después de producirse el primer desembarco francés en el territorio - tenemos datada su presencia en la capital argelina desde mayo de 1832- (1), permaneciendo allí durante más de treinta años, primero como agente comercial de España y más tarde como cónsul general. De origen vasco, era un profesional altamente cualificado, agudo observador y muy capaz, como se evidencia en sus interesantes despachos a Madrid (2). Insustituible en Argel por su conocimiento del país y excelentes relaciones personales con la administración colonial, sirvió eficazmente a España y a sus connacionales, tanto residentes como inmigrados (3), por lo general pobres jornaleros analfabetos a quienes defendió contra la explotación de patronos desaprensivos, sin olvidarse de los numerosos

(1) A. H. N. Estado (Argelia), leg. $6.153^{2}$.

(2) Vid. por ejemplo el muy notable remitido en marzo de 1862 sobre la coyuntura económica argelina de los primeros años 60 , publicado y estudiado en VILAR, Juan Bta.: Transformations agraires algériennes au milieu du XIX $X^{a}$ siècle et crisi economique du 1861, selon un memoire du Consulat d'Espagne a Alger. Revue d'Histoire Magherebine, n. $19-20$ [Tunis, 1980), pp. 185-197. [Traducción de Míkel de Epalza.]

(3) VILAR, J. B.: Emigracioón española a Argelia (1830-1900). Colonización hispánica de la Argelia francesa. Madrid. 1975. pp. 52-157. 
refugiados políticos que por entonces solían buscar asilo en Argelia, y a quienes no dudaba en brindar ayuda y protección (4).

Por el contrario, de Carlos Vidal apenas sabemos nada. Su paso por el consulado de Argel resultó efímero, dado que si bien fue confirmado en el cargo, no tardó en ser removido del mismo por la triunfante revolución de septiembre de 1868 . En la memoria aquí estudiada, Vidal prueba ser competente funcionario y además buen conocedor de la realidad argelina, que analiza con ponderación, acierto y clarividencia.

Una Real cédula de 1857, remitida por el Gobierno español a sus representantes en el extranjero, había hecho preceptivo el envío a Madrid de anuales memorias comerciales desde cada uno de los distritos consulares. Vidal remitirá puntualmente la suya - febrero 1866 - referida al año anterior, no obstante llevar pocos meses en el cargo. Tal diligencia es encomiable habida cuenta de las dificultades que representaba reunir rápidamente una información compleja y fidedigna en momentos en que la moderna ciencia estadística daba sus primeros pasos. Esa información era muy necesaria en España como indicativo cierto del estado del tráfico mercantil con Argelia, contemplado en el marco de los intercambios franco-españoles, impulsados por el nuevo tratado comercial con Francia (5).

El comercio español con Argel, pletórico de posibilidades, a la sazón conocía momentos esperanzadores. Con una tradición secular que se remontaba a la fase precolonial (6) y reactivado en los primeros tiempos de la ocupación francesa (7); iniciada la década de 1860 , todavía detentaba un destacado segundo lugar en cuanto a número de buques entrados en puerto -326 en 1861 - , si bien, apenas representaban un 10,21 por ciento del valor de las mercancías dado el escaso tonelaje de las embarcaciones utilizadas (8). La imposición de cuatro francos por tonelada a los navíos españoles, de acuerdo con los aranceles aplicados en Argelia precisamente desde 1861 (9), incidiría negativamente sobre un tráfico ya declinante.

La memoria de Vidal presenta tres partes diferentes, aunque no siempre bien delimitadas: el estado general de la navegación en Argelia, el movimiento del puerto de Argel, y el tráfico hispano-argelino por ese puerto. Respecto al primer punto, el cón-

(4) Un buen ejemplo, en su generosa conducta es el caso del grupo de emigrados progresistas huidos a Argel desde Carta. gena en 1844. Vid. VILAR, J. B.: Mutaciones ideológicas y empresas colonialistas de un emigrado polltico murciano. Valle de San Juan de Argelia (1844-1845). Murgetana, 59, (Murcia, 1980), pp. 83-113.

(5) Vid. BECKER, Jerónimo: Relaciones comerciales entre España y Francia en el siglo XIX. Madrid 1910 . Precisiones útiles en PLAZA PRIETO, J.: El desarrollo del comercio exterior español desde principios del siglo XIX a la actualidad.

(6) VILAR, J. B.: Ralaciones comerciales hispano-argelinas en el periodo 1791-1814. Hispania, XXXIV (Madrid, 1974), pp 435-442; VILAR, Relaciones diplomáticas y comerciales hispano-argelinas en las postrimerlas de la Argelia otomana 118 i41830). Hispania XXXVI 119761, pp. 623-638; VILAR, Los judlos de Argel, Orán y Gibraltar, intermediarios del trafico hispanoargelino entre 1791 t 1830: Asunto Bacri, Miscelánea de Estudios Arabes y Hebráicos, 24, facs. 2 (Granada, 1975 l, 0 p 67-73; VILAR, Fernando VII, la Inquisición y los judios de Gibraltar. Mague, 33 (Caracas, 1973), pp. 6-10; núm. 34, pp. 10-13; VILAR, Sociedad española en la Argelia otomana 17791-1814). Almenara, 9 (Madrid, 1976), pp. 63-72; CARA DEL AGUILA, Louis: Les espagnols en Afrique. Les relations politiques et commerciales avec la Regence d'Alger. Bordeaus, 1974: EPALZA, Míkel de: Algunas consecuencias del tratado de paz hispano-argelino de 1786 . Homenaje a Guillermo Guastavino (Madrid, 1974), op. 443-449; EPALZA, Intereses arabes e intereses españoles en las paces hispano. musulmanas del siglo XVIII. Anales de Historia Contemporánea, 1 (Murcia, 1982), pp. 7-17; EPALZA, Los Soler menor. quines en el Mediterráneo islámico (Magrebi-Oriente) y la expansión magrebi de los menorquines. Sigios XVih-XIX. Revista de Menorca, LXXI (Mahón, 19801, pp. 106-112; VILAR, J. B.: Menorca y el rescate de cautivos españoles en Argel por la migón Ortiz de Zugasti en 1827. Revista de Menorca (1985). En prensa. Sobre esta temática y período inciden, a su vez, los profesores I. H. TERKI, N. MALKI y H. ARAAF, de la Universidad de Orán, con sus tesis doctorales en curso de realización.

(7) VILAR, J. B.: Le commerce espagnol avec I'Algérie au debut de la periode coloniale. Revue d'Histoire Maghrebine, 12 (Tunis, 1978), pp. 289-297 (reproducido con incorporación de apéndices originales en el Bulletin de la Societé de Géographie et Archeologie d'Oran, an 1977-1978]; VILAR, Alicante en el tráfico espaffol con Argelia (1830-1833). Revista del instituto de Estudios Alicantino, 22 (1978), pp. 75-90. Para el periodo posterior a 1833 cuantiosa información sobre el comercio hispalo-argelino en VILAR. Emigración española a Argelia..., pp. 147-153, 205-211, 401.479.

(8) Vid. apéndice documental.

(9) RAPPORT sur le droit de tonnage et sur diverses autres questions concernant le régime commercial de l'Algérie. Alger 1861 , pp. 15-31. 
sul traza un sucinto panorama en el marco de los tratados de comercio vigentes entre Francia y otros países, con particular mención de aquéllos en que se hace expresa referencia al caso de Argelia. En la información aportada se presiente la negativa incidencia sobre el comercio exterior argelino de las crisis cíclicas de origen agrario conocidas por el territorio en los últimos años (10), coetáneas de las estudiadas por $N$. Sánchez Albornoz en el caso de España (11).

Apunta Vidal que el cabotaje entre los puertos argelinos era realizado por buques extranjeros por encima de las 80 toneladas, pero bajo pabellón francés y con sujeción a importantes gravámenes fiscales. Ésta era la conocida como "Marina africana», que en 1863 estaba constituida por 70 buques, en su mayoría italianos, seguidos a considerable distancia por otros españoles -28 en total-, en tanto los franceses eran sólo cuatro (12). Los británicos - casi siempre malteses y gibraltareños - , tunecinos, marroquíes, portugueses, holandeses, suecos, austro-húngaros y alemanes, entre otros (13), frecuentaban también aquellas aguas.

Algo parecido acontecía con la pesca en las costas de Argelia, actividad productiva dominada también por italianos, salvo en el litoral oranés donde la presencia española resultaba determinante. La pesca del coral continuaba siendo una especialidad de los marineros llegados de Cataluña, Baleares y de diferentes puntos de Italia. En 1862, sobre un conjunto de 239 pesqueros que operaban en las costas argelinas, apenas se computaban cuatro de matricula francesa (14).

TABLA I

MOVIMIENTO DEL PUERTO DE ARGEL EN 1865

\begin{tabular}{|c|c|c|c|c|c|}
\hline CONCEPTO & BANDERA & BUQUES & $\mathrm{TM}$ & $\begin{array}{l}\text { TRIPULANTES } \\
\text { Y PASAJEROS }\end{array}$ & $\begin{array}{l}\text { VALOR DEL CARGO } \\
\text { (En escudos) }\end{array}$ \\
\hline \multirow{4}{*}{ Entradas } & Española ............ & 423 & 15.449 & 2.869 & 263.329 \\
\hline & Sueca y noruega... & 25 & 7.300 & 293 & 325.309 \\
\hline & Italiana................ & 31 & 2.626 & 279 & 73.703 \\
\hline & Británica............ & 14 & 2.816 & 114 & 37.268 \\
\hline \multirow{4}{*}{ Salidas } & Española ........... & 422 & 15.459 & 2.899 & 647.560 \\
\hline & Sueca y noruega... & 26 & 7.770 & 308 & - \\
\hline & Italiarla............. & 23 & 2.337 & 226 & 87.777 \\
\hline & Británica............. & 14 & 2.816 & 114 & 29.070 \\
\hline
\end{tabular}

(10) VILAR, Transformations agraires algériennes..., pp. 185-197. Vid., también VILAR, La coyuntura argelina de 1866, a través de un informe confidencial español. Anales de Historia Contemporánea, 1 (Murcia, 1982), pp. 119-150. El con texto histórico del fenómeno estudiado nos viene dado por los numerosos y excelentes estudios de Xavier Yacono sobre Argelia durante el II Imperio, todos ellos de ineludible consulta. En particular: YACONO, La colonisation des plaines du Chélif (De Lavigérie au confluent de la Minal. Alger 1955-1956, 2 vol. Vid. también JULIEN, CH. A.: Histoire d'Algérie Contemporaine: la conquête et les débuts de la Colonisation: 1827-1871. París 1964

(11) SANCHEZ-ALBORNOZ, Nicolás: España hace un siglo: una economla dual, 2. ed. Madrid, 1977; SANCHEZ.ALBORNOZ. Jalones en la modernización de España. Barcelona, 1975, pp. 7-13, 99-146.

112) Vid. apéndice documental. Información adicional en AHN, Estado, legs. 6.150², 6. 154 y 6. 160; AMAE, Correspondencia (Argel), leg. 1.818

13) Una navegación, por cierto, muy semejante a la registrada en puertos españoles. Vid. VILAR, J. B.: El esfuerzo industrializador, en vol. XIV de "Historia General de España y America" (La España liberal y romántica, 1833-1868). Madrid, Rialp, 1983, pp. 114-216.

114) Apéndice documental. 
La navegación extranjera con el puerto de Argel era fundamentalmente española, nórdica, italiana y británica, por ese orden. Vidal aporta (ver tabla I) un cuadro pormenorizado del movimiento de buques, con expresión del número de embarcaciones, tonelaje, tripulaciones y pasajeros, y valor de los cargamentos tanto a la entrada como a la salida.

De la tabla anterior se infiere que la navegación extranjera en el puerto de Argel estaba en manos de españoles y nórdicos en cuanto al volumen de mercanclas. Pero en tanto la balanza comercial era altamente deficitaria para España, los escandinavos, colocadas sus mercancías, marchaban en lastre "con las ventajas que les proporciona la franquicia de que disfrutan los cargamentos de maderas de construcción, que es lo que generalmente traen" (15). El comercio italiano con Argel era también deficitario, en tanto a los británicos les resultaba favorable el escaso tráfico realizado con la capital argelina.

Noruegos y suecos vendían en Argelia, aparte de la mercancía ya mencionada, hierro y acero. Los italianos transportaban a la colonia francesa subsistencias perecederas - fruta, legumbres secas, queso, carne salada, harina, arroz - , así como sebo y duelas. En cuanto a los ingleses, hierro, acero, carbón, minerales, material ferroviario, pero también tabaco, queso y concha. La navegación francesa, sin duda la más importante, pero cuya cuantía y cargamentos no se especifican, nos consta por otras fuentes (16) que introducía por Argel - desde Marsella y puertos mediterráneos principalmente - cuanto era necesario para el equipamiento y subsistencia del territorio; desde maquinaria y manufacturas a artículos de primera necesidad. Exceptuada España, a cuya presencia mercantil en la posesión francesa me referiré seguidamente, los restantes países itienen una parte insignificante en el comercio de esta colonia" (17).

Vidal (ver tabla II) aporta la serie completa de buques españoles entrados en Argel en los últimos veinticinco años.

El máximo número de embarcaciones corresponde a 1842, año en que a consecuencia del último levantamiento musulmán registrado en el distrito de Argel, quedaron cortadas las comunicaciones de la capital con el interior, haciéndose necesario aprovisionarla por mar.

Hasta 1848 la navegación española se mantuvo en un buen nivel, pero la revolución de ese año en la metrópoli, y el incierto futuro de la colonia durante la II República y en la fase inicial del II Imperio, determinaron una sustantiva contracción del tráfico. No fue reactivado hasta 1854, en que la crisis vitícola en Francia e Italia, determinó la masiva importación de caldos españoles, alcanzando el tráfico español su techo en 1861. Ahora bien, el recargo arancelario establecido en ese año sobre buques extranjeros, y un nuevo incremento fiscal introducido en el 63, derechos estos últimos de que Vidal da noticia pormenorizada en su informe, representarían una seria rémora a la expansión de la tradicional corriente comercial mantenida por España con Argelia.

El tráfico hispano-argelino era realizado casi exclusivamente por buques españoles, siendo deficitario para la primera. En el caso de los intercambios con Argel, en

\footnotetext{
(15) Ibidem.

(16) AHN, Estado, leg. 6.150², 6.154,6.160, 8.357; AMAE, Correspondencia (Argell, legs, 1.818, 1.819. Vid. también CES-CAUPENME, Alfredo: La España y la Argelia a propósito de la Exposición internacional franco-española. Argel, 1864 , pp. 5-84; CARRERAS, M.: Curso de Geografía y 'Estadistica industrial y comercial. Madrid, 1866, pp. 96-138.

(17) Apéndice documental.
} 
TABLA ॥

BUQUES ESPAÑOLES ENTRADOS EN ARGEL (1840-1864)

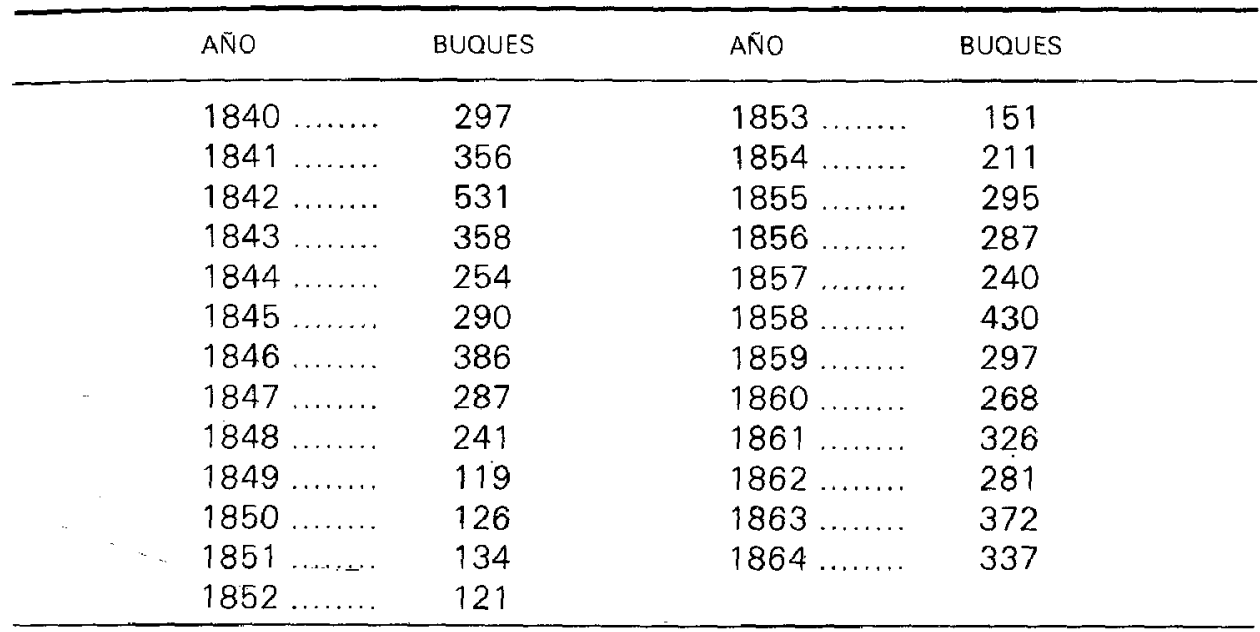

Fuente: AMAE. Correspondencia (Argel), leg. 1819

1865 el valor de las exportaciones argelinas duplicaron ampliamente el de los artículos importados de España, 722.821 escudos frente a 356.443. La cuantía y signo de este comercio era aproximadamente igual que en el año precedente, lo que parece indicar que la balanza de pagos se mantenia permanentemente adversa para España, hecho que por lo demás no era nada nuevo en la historia de los intercambios comerciales entre ambos países, incluso desde antes de la ocupación francesa (18).

Al frente de las exportaciones españolas a Argel figuran los vinos y aguardientes alicantinos, mallorquines y catalanes, el esparto y pimiento molido murcianos, las legumbres y frutas frescas y secas que, así como el aceite, el queso y el ganado porcino, procedian preferentemente de Baleares y reino de Valencia. Por último, "piedras de Menorca» para la construcción (19). Como puede verse, productos alimentarios principalmente, que palmesanos, menorquines y levantinos introducían con sus frágiles barcas en la populosa urbe norteafricana.

Entre las exportaciones argelinas con destino a Baleares y la Península en 1865 figurarán en primer lugar el ganado vivo. Exactamente 50.235 cabezas de bovino $y$ ovino, adquiridas a buen precio. Le segulan en importancia el salvado y, dato curioso, harina de trigo con destino al avituallamiento de las islas Canarias. El carbón vegetal era tomado por aquellos buques que, careciendo de carga de retorno, no deseaban regresar en lastre. Las restantes partidas resultan irrelevantes.

Los buques españoles que hacian la singladura con Argel - 11 con 423 entradas en 1865 - eran pequeñas embarcaciones entre 20 y 40 toneladas: laúdes, jabe-

(18) Vid. notas 6 y 7

(19) Apéndice documental. 
ques, faluchos, místicos y polacras de uno o dos palos. Raras veces tenían tres palos y alcanzaban las 60 toneladas, y sólo en ocho casos sobrepasaban ese tope, pero sin llegar a las cien toneladas. Su procedencia era la siguiente:

\begin{tabular}{|c|c|}
\hline PUERTO DE MATRÍCULA & N. BUQUES \\
\hline Palma de Mallorca ................ & 42 \\
\hline 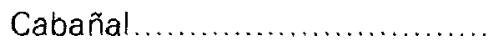 & 22 \\
\hline Santa Pola................... & 14 \\
\hline Mahón v Ciudadela ............... & 12 \\
\hline Torrevieja ......................... & 6 \\
\hline Altea ............................. & 5 \\
\hline 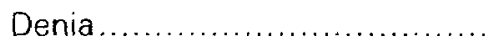 & 4 \\
\hline 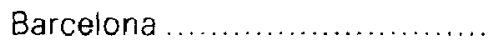 & 3 \\
\hline San Felíu, Arens y Tarragona... & 3 \\
\hline 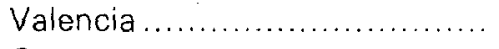 & 3 \\
\hline Cartagena........................ & 2 \\
\hline Castellón ......................... & 2 \\
\hline Ibiza ............................. & 2 \\
\hline 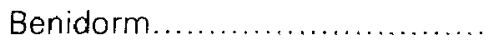 & 1 \\
\hline Villajoyosa .......................... & 1 \\
\hline
\end{tabular}

Fuente: AMAE. Correspondencia (Argel), leg. 1819

Como puede verse, en la fase final del reinado de Isabel ll la navegación palmesana, y mallorquina en general, controlaba el panorama del tráfico español con Argel. después de haber logrado imponerse a los menorquines, hegemónicos en los primeros tiempos de la colonia (20).

Corresponde un destacado segundo lugar en ese tráfico a la región valenciana, pero más en razón de la proyección sobre Argelia de modestas localidades litorales, vinculadas mercantilmente a este país desde siempre, como Santa Pola, Torrevieja, Altea. Denia, Villajoyosa o Benidorm, que por el tráfico realizado desde grandes y medianos puertos como Valencia, Castellón o Alicante. La navegación catalana, muy importante en otra época, continúa presente en aquellas aguas, en tanto la de Cartagena es meramente testimonial, por atraer sus preferencias la región de Orán, muy próxima a las costas murcianas.

\section{Abreviaturas utilizadas}

AHN: Archivo Histórico Nacional (Madrid).

AMAE: Archivo del Ministerio de Asuntos Exteriores (Madrid).

(20) VILAR, J. B. Las Baleares y la expedición francesa a Argel en 1830. Mayurqa, núm. 13 (Palma de Mallorca, 1975), pp. 220-225; VILAR, Le commerce espagnol avec l'Algèrie... Op. cit. 


\section{APENDICE DOCUMENTAL (1)}

Exposición razonada del movimiento mercantil del puerto de Argel y de los de la demarcación de este consulado general con los puertos de la España peninsular y ultramarina.

El movimiento mercantil de los puertos de España con los de Argelia se efectúa por lo general con la bandera española. El estado adjunto, señalado con la letra $A$, demuestra el número de buques que han entrado y salido en el puerto de Argel y demarcación consular en todo el año de 1865 y el valor de la importación y exportación que, comparado con el resultado del año 1864, no presenta aumento ni disminución notable.

El valor de la importación procedente de España es, en 1865, de 366.378 escudos, y el de la exportación con destino a puertos españoles asciende a escudos 722.821. De modo que dichas operaciones mercantiles dan un resultado en metálico de 356.443 escudos a favor de este país.

Por la nota adjunta, letra $B$, se demuestra la clase de géneros que se exportan de Argel con destino a España, y las cantidades de los que se han embarcado en todo el año 1865.

El artículo más rico y de entidad es el ganado, resultando exportadas desde el puerto de Argel en dicho año con destino a la Península e islas Baleares 50.232 cabezas a precios muy convenientes, pues los corrientes en estos mercados son el de 40 a 60 escudos por cada buey y el de 6 por carnero. El de las vacas y terneros varia mucho según su clase y edad. El trigo y harina suelen llevarse a Canarias con nuestra bandera, como se nota en el estado número 1. Sus precios son de 9 a 10 escudos los cien kilogramos, según la clase de la harina.

Algún carbón vegetal se exporta para España, pero sólo lo toman los patrones por no regresar en lastre. El salvado se embarca en cantidad regular. Su precio es, por cien kilogramos, 2 escudos y medio. Los demás artículos se exportan con nuestra bandera en cortas cantidades.

La navegación con bandera española presenta una variación algo notable en el número de buques entrados en el puerto de Argel en diversos años anteriores. Las causas de su aumento y disminución son suficientemente conocidas como se demuestra a continuación:

$\begin{array}{cccc}\text { Año } & 1840 & \text { entrados } & 297 \\ " & 1841 & " & 356 \\ " & 1842 & " & 531 \\ " & 1843 & " & 358 \\ " & 1844 & " & 254 \\ " & 1845 & " & 290 \\ " & 1846 & " & 386 \\ " & 1847 & " & 287 \\ " & 1848 & " & 241 \\ " & 1849 & " & 119 \\ " & 1850 & " & 126 \\ " & 1851 & " & 134\end{array}$

(1) AMAE, Correspondencia (Argell, leg. 1.819. 


$\begin{array}{cccc}\text { Año } & 1852 & \text { entrados } & 121 \\ " & 1853 & " & 151 \\ " & 1854 & " & 211 \\ " & 1855 & " & 295 \\ " & 1856 & " & 287 \\ " & 1857 & " & 340 \\ " & 1858 & " & 430 \\ " & 1859 & " & 297 \\ " & 1860 & " & 268 \\ " & 1861 & " & 326 \\ " & 1862 & " & 281 \\ " & 1863 & " & 372 \\ " & 1864 & " & 337\end{array}$

En el año 1842 resultan entrados 531 buques españoles. Esto fue a consecuencia de la gran sublevación dé los árabes que impedía la entrada de víveres por tierra, siendo los buques españoles los que, en aquella época, abastecían la plaza de Argel.

Del año 1849 al 1853 , se disminuyó considerablemente la navegación española por efecto de la paralización del comercio y la desconfianza que produjo en Argelia la revolución de Francia, época en que se habló del abandono de esta colonia. El aumento notado desde el año 1854 lo produjo la gran importación de vinos españoles para suplir la talta de este líquido por causa de la enfermedad de las viñas en otros países, habiendo continuado luego dicho aumento por la mucha exportación de ganado.

Los derechos de tonelada, puerto, pilotaje, y sanidad que pagan en la Argelia los buques extranjeros no dejan de presentar algunas trabas a la navegación española, particularmente sobre la importación. La ley vigente de 9 de mayo de 1863, sobre derechos de tonelada prescribe lo siguiente:

1. El derecho de tonelada, actualmente impuesto a los buques extranjeros en los puertos de la Argelia se percibirá por las toneladas de flete sobre las mercancias que dichos buques desembarquen o embarquen.

2: El derecho de tonelada se percibirá también en proporción al número de pasajeros embarcados o desembarcados, contando una tonelada por cada uno de ellos. Los niños, de cualquier edad que sean, cuentan por un pasajero. Un caballo por dos toneladas. Un carruaje de dos ruedas por tres, y de más de dos ruedas por cuatro.

Los derechos que se citan son los siguientes:

Por pilotaje de entrada, por cada tonelada ............... frs 0,11 ctms.

Por pilotaje de salida, por cada tonelada.................. frs 0,55 ctms.

Por sanidad .......................................... frs 0,10 ctms.

Por pasaporte de aduana, cada buque .................. frs 1,25 ctms.

Por tonedada ............................................ frs 4

La aplicación de dichos derechos se efectúa como sigue:

Cada pasajero representa una tonelada, según ,queda dicho. Por consiguiente, si un buque de 100 toneladas llega con 40 de cargo y 40 pasajeros paga en razón de 80 toneladas. Pero si el cargo y pasajeros, comprendidos los que embarcan en su salida, componen un número mayor del que realmente mide el buque, en este caso paga sólo por las 100 toneladas de su verdadera cabida. Los buques que no llegan al porte de 25 toneladas no están sujetos al derecho de pilotaje.

Los que entran de arribada forzosa se hallan exentos del derecho sanitario. El buque que llega con cargo completo, que desembarca y sale también con cargo, paga los derechos por entero sobre su cabida. 
El que llega en lastre y carga 14/15 de cabida en géneros del país, para tan sólo los derechos de pilotaje y sanidad, aunque lleve pasajeros. Si su cargo no llega a los citados 14/15 de su cabida, paga el derecho de tonelada del cargo que embarca, y el de pilotaje, sanidad y demás en razón de las que mida el buque.

Si llega con cargo y sale en lastre paga el derecho de tonelada sobre las que representa el cargo que desembarca, y el pilotaje y sanidad sobre el total de las que mida el buque. Si el buque llega con cargo menos de 1/20 de su cabida, se considera en lastre. Cargando géneros del país en cantidad menor de 14/15 paga el derecho de tonelada sobre las que representa el cargo que embarca, pero el derecho de sanidad y pilotaje lo paga en razón del porte del buque.

La aplicación de los citados derechos demuestra evidentemente la idea de favorecer la exportación de géneros del país. Un buque de vela o vapor español que viniese a Argel con 100 toneladas de cargo y 50 pasajeros, suponiendo su porte de 200 toneladas, pagaría por las 150 que representa el cargo y pasajeros 600 francos. Más 22 por derechos de pilotaje de entrada, 10 de salida, 20 de sanidad, 1,25 por pasaporte de aduana y 20 de corredor. En total 673,25 francos.

Esto se entiende saliendo en lastre. Si cargase, crecería el gasto en proporción al cargo y pasajeros que embarcase, teniendo presente además que debería satisfacer unos 130 francos por derechos de Consulado.

El mismo buque, entrando en lastre sin pasajeros y cargando más de $14 / 15$ de su porte en productos del país, aunque llevase pasajeros, pagaría tan sólo los derechos de pilotaje y sanidad. Pero como en su viaje no podría hacer más operación de comercio que la que efectuase a su salida de Argel, en un buque de gran porte el flete no correspondería a sus gastos de venida y vuelta, razón por la cual el movimiento mercantil con bandera española se efectúa en la Argelia en buques pequeños. Como se demuestra a continuación.

Las entradas de buques españoles en Argel ha sido, en todo el año 1865, en número de 423 . Dichas entradas se han efectuado con los 122 buques siguientes:

42 de la matrícula de Palma.

22 del Cabañal.

14 de Santa Pola.

12 de Mahón y Ciudadela.

2 de Cartagena.

6 de Torrevieja.

3 de Barceiona.

1 de Benidorm.

4 de Denia.

3 de San Feliu, Arens y Tarragona.

3 de Valencia.

2 de Castellón.

1 de Villajoyosa.

5 de Altea.

2 de Ibiza.

La mayor parte de estos buques miden de 20 a 40 toneladas. Muy pocos de 50 a 60. Ocho pasan de 60 y ninguno llega a 100 .

La bandera española trae a Argel vino, aguardiente, frutas secas y frescas, esparto, pimiento molido, legumbres secas, piedras de Menorca, cerdos, aceite y queso.

La bandera sueca importa maderas de construcción, hierro y acero.

La inglesa, hierro, carbón fósil, acero, material para ferrocarriles, tabaco, queso, concha y mineral. 
La italiana, frutas, queso, legumbres secas, harina, arroz, carne salada, sebo y duelas.

La bandera francesa trae exclusivamente los artículos de industria y productos de su país necesarios en el consumo de la Argelia.

Las demás naciones tienen una parte insignificante en el comercio de esta colonia.

La importación y exportación del puerto de Argel con las cuatro citadas banderas en el año 1865 ha sido la siguiente:

TABLA

\begin{tabular}{|c|c|c|c|c|c|}
\hline CONCEPTO & BANDERA & BUOUES & $T M$ & $\begin{array}{l}\text { TRIPULANTES } \\
\text { Y PASAJEROS }\end{array}$ & $\begin{array}{l}\text { VALOR DEL CARGO } \\
\text { (En escudos) }\end{array}$ \\
\hline \multirow{4}{*}{ Entradas } & Española. & 423 & 15.449 & 2.869 & 263.329 \\
\hline & Italiana............... & 31 & 2.626 & 279 & 73.703 \\
\hline & Inglesa ............... & 14 & 2.816 & 114 & 37.268 \\
\hline & Sueca y noruega... & 25 & 7.300 & 293 & 325.309 \\
\hline \multirow{4}{*}{ Salidas } & Española ........... & 422 & 15.459 & 2.899 & 647.560 \\
\hline & Italiana............. & 23 & 2.337 & 226 & 87.777 \\
\hline & Inglesa.............. & 14 & 2.816 & 114 & 29.070 \\
\hline & Sueca y noruega... & 26 & 7.770 & 308 & - \\
\hline
\end{tabular}

Fuente: AMAE. Correspondencia (Argel), leg. 1819

Resulta del anterior estado que España e Italia han exportado a Argel por valor mayor que el de su importación. Para Inglaterra ha sido mayor su exportación, Suecia y Noruega han desembarcado y vendido sus mercancias saliendo luego en lastre, con las ventajas que les proporciona la franquicia de que disfrutan los cargamentos de maderas de construcción, que es lo que generalmente traen.

Entre los tratados de comercio y navegación que la Francia ha estipulado con diversas naciones y que hacen particular mención de la Argelia, se anotan el estipulado con Rusia en 14 de junio de 1857, el de Inglaterra de 23 de junio de 1860 , el de Bélgica de 1 de mayo de 1861, y la convención de navegación con Italia de 13 de junio de 1863. Todos favorecen a las banderas de dichas naciones.

El cabotaje en la Argelia se reservó a la bandera francesa. Pero no aprovechando ésta el privilegio, se creó una marina mixta que llaman africana. Fueron admitidos en ella los buques extranjeros de ochenta o más toneladas, tomando bandera francesa pero conservando sus tripulaciones extranjeras, debiendo pagar el derecho de 40 francos por tonelada por solo una vez y el debido a la caja de inválidos de Francia.

Esa institución favoreció la navegación de las costas, así como la emigración y deserción de muchos marineros españoles. En 1863, la marina africana contaba con 70 buques y tenía a su disposición 216 patrones, de los cuales 28 eran españoles, 4 franceses, 16 africanos y 168 italianos, con más de ochocientos marineros, muchos de ellos españoles. Desde entonces no ha habido variación notable en dicha marina.

La pesca en las costas de Argelia la ejercen en primer lugar los italianos, pues 
son muchisimos los buques de dicha nación que se dedican a ella. La del coral se efectúa exclusivamente por españoles e italianos, y la hacen por las costas del Este de la Argelia. En 1862, se ocuparon en dicha pesca 239 buques, de los cuales sólo cuatro eran franceses.

Los puertos de las provincias de Argel y Constantina en que se nota más movimiento comercial con bandera española son en primer lugar el de Argel, y después las de Bugía, Philippeville, Bona, Cherchell y Túnez. En el resto de la Argelia los de Orán y Mostaganem.

La bandera italiana hace su principal comercio al Este de la provincia de Argel en La Calle, Philippeville y Bona. Inglaterra y Suecia con los puertos de Argel y Orán.

El comercio de España con esta colonia francesa, si bien no es hoy de grande importancia, la circunstancia de hallarse establecidos en la Argelia más de $\mathbf{4 0 . 0 0 0}$ españoles es digna de consideración. Sería de desear que el gobierno francés concediese a la bandera española disminución del derecho de tonelada sobre el cargo, supresión del impuesto sobre los pasajeros que conduce y autorización para ejercer el comercio de cabotaje.

Argel, 28 de febrero de 1866. El Cónsul general interino. Carlos Vidal.

Excmo. Sr. Primer Secretario de Estado. Madrid. 